\title{
Ungleiche Paare: Kasuswechsel in koordinierten Nominalphrasen
}

Książka BAstiana Sicka Der Dativ ist dem Genitiv sein Tod zainspirowała szczegółowe badania nad używaniem przypadków we współczesnym języku niemieckim. SICK (2004) opisał tylko pół prawdy (albo jeszcze mniej), ponieważ obserwujemy nie tylko zastępowanie dopełniacza przez celownik, ale też zastępowanie celownika przez dopełniacz; ponadto obserwujemy zastępowanie celownika przez biernik i biernika przez celownik; poza tym wszystkie przypadki bywają zastępowane przez mianownik. Wszystko to występuje obficie w apozycjach, ale też w rekcji czasowników i przyimków, na przykład Er begab sich in einem anderen Raum, chociaż in w określeniach kierunku wymaga biernika: Er begab sich in einen anderen Raum (dosł. „udał się w inny pokój“). Zaskakujące jest jednak to, że taka zmiana przypadków zdarza się też we frazach nominalnych współrzędnych, jak na przykład den Leichen von Generalbundesanwalt Buback und seines Fahrers (zamiast: seinem Fahrer). W niniejszym artykule prezentuję $\mathrm{i}$ interpretuję wybrane przykłady takiej zmiany przypadka („,zmiana“ zarówno w sensie synchronicznym jak i diachronicznym), dokumentując $\mathrm{w}$ ten sposób wzrastającą niepewność rodzimych użytkowników języka niemieckiego co do użycia przypadków. Można przypuszczać, że system niemieckich przypadków przeobraża się lub nawet podlega rozpadowi.

In gründlichen Untersuchungen zum Kasusgebrauch im gegenwärtigen Deutsch, ausgelöst durch BASTIAN SiCKs Buch Der Dativ ist dem Genitiv sein Tod, habe ich festgestellt, dass SICK (2004) nur die halbe Wahrheit (oder noch weniger) beschreibt, da nicht nur der Genitiv dem Dativ weicht, sondern zunehmend auch Dativ durch Genitiv ersetzt wird. Dazu werden Dativ und Akkusativ vermischt und für alle drei obliquen Kasus tritt oft der Nominativ ein. All das betraf vorwiegend den Kasusgebrauch in Appositionen, aber auch in der Verb- und Präpositionsrektion, wo man neuerdings etwas findet wie Er begab sich in einem anderen Raum, obwohl in als Richtungsangabe den Akkusativ fordert. Mittlerweile stellte ich mit Überraschung fest, dass Kasuswechsel auch in koordinierten NPs zu finden ist, wo z.B. von den 
Leichen von Generalbundesanwalt Buback und seines Fahrers (statt: seinem Fahrer) die Rede ist. In diesem Aufsatz werden weitere Belege für Kasuswechsel (im synchronen und diachronen Sinne) in Koordinationen vorgestellt und interpretiert, die zunehmende Unsicherheit deutscher Sprecher im Kasusgebrauch zeigen und vermuten lassen, dass sich das Kasussystem des Deutschen in Veränderung, möglicherweise im Verfall befindet.

In detailed investigations, caused by BASTIAN SicK’s book Der Dativ ist dem Genitiv sein Tod, I discovered that the statements of SICK (2004) present only half the truth (or even less), since the replacement of the genitive by the dative is only part of a more extensive change: the dative is also replaced by the genitive (especially after prepositions), dative and accusative are exchanged, and all three oblique cases are exchanged by the nominative. My initial findings concerned cases assigned by verbs and prepositions as well as cases in appositives. Meanwhile, I noticed that you can find case change also in conjoined NPs like den Leichen von Generalbundesanwalt Buback und seines Fahrers (rather than: seinem Fahrer). In this article many examples for case change (in its synchronic as well as in its diachronic reading) will be presented and interpreted. They show the increasing uncertainty of speakers of German in their use of cases. We can draw the conclusion that the case system of German is going to be changed and possibly given up altogether.

\section{Kasuswandel im gegenwärtigen Deutsch}

Bastian Sicks Buch Der Dativ ist dem Genitiv sein Tod (SICK 2004), das 2006 bereits in 29. Auflage erschien, regte mich zu Untersuchungen des Kasusgebrauchs im gegenwärtigen Deutsch an. Der Buchtitel macht von der umgangssprachlichen Dativumschreibung des Genitivs Gebrauch (vgl. meinem Vater sein Haus) und suggeriert, dass der Dativ im Deutschen zunehmend den Genitiv ersetzt. Was Sick jedoch präsentiert, stellt sich als nur halbe Wahrheit heraus - oder eher ein Viertel der Wahrheit: Untersuchungen zur Rektion von Präpositionen von Di MEOLA (2000) zeigen bereits, dass auch umgekehrt der Dativ durch den Genitiv ersetzt wird. So werden die traditionell den Dativ regierenden Präpositionen trotz, dank, entgegen, entsprechend und samt mittlerweile überwiegend mit Genitiv gebraucht. Und meine eigenen Untersuchungen ergaben, dass auch bei Verben der Dativ oft durch den Genitiv ersetzt wird. Seit langem werden zudem in der norddeutschen Umgangssprache, mittlerweile auch im Standarddeutschen, der Dativ durch den Akkusativ und der Akkusativ durch den Dativ ersetzt. Neuerdings findet sich sogar die Ersetzung des Akkusativs durch den Genitiv, jedenfalls in der Wendung wider besseren Wissens. In einem Leserbrief an den Kölner Stadtanzeiger vom 2.2.2008 schlägt HUPPERTH (scherzhaft) vor: 
Ungleiche Paare: Kasuswechsel in koordinierten Nominalphrasen

Nachdem sich die Formulierung wider besseren Wissens statt wider besseres Wissen auch bei den Gralshütern der korrekten deutschen Sprache, den Redakteuren der Presseagenturen und Journale, durchgesetzt hat, schlage ich zeitgemäß der Karnevalsgesellschaft Aachen vor, ihren Orden umzutaufen in Wider des tierischen Ernstes. ${ }^{1}$

Zudem werden die drei obliquen Kasus durch Nominativ ersetzt (vgl. 2.6), offenbar Anzeichen für die Tendenz, den Nominativ wegen seiner Endungslosigkeit als ,Nennform‘ anstatt der obliquen Kasus zu benutzen (vgl. WIEDENMANN 2004), d.h. über seine Funktionen als Subjekt und Prädikativ hinaus. Ergo: Alle Kasus des Deutschen werden zunehmend miteinander vermischt. In meinen Untersuchungen zum Kasusgebrauch im heutigen Deutsch (vgl. VATER 2007) stellte ich fest, dass sowohl in losen Appositionen als auch in der Kasusrektion nach Verben und Präpositionen große Veränderungen zu beobachten sind. ${ }^{2}$ Besonders überrascht war ich, solche Veränderungen auch in einem Bereich zu finden, den ich für besonders stabil hielt: im Kasusgebrauch in Koordinationen. Im Folgenden werden genügend Beispiele angeführt, die zeigen, dass auch in diesem Bereich große Unsicherheit eingetreten ist.

Es ist wichtig, zwischen Änderungen im Kasusgebrauch und Änderungen in der Kasusform zu unterscheiden. Bei der Kasuswahl geht es darum, welchen Kasus ein Verb, ein Adjektiv oder eine Präposition regiert bzw. welcher Kasus in Appositionen oder koordinierten Nominalphrasen (NPs) verwendet wird. ${ }^{3}$ Hier geht es beispielsweise um den Wechsel vom Genitiv zum Dativ, vom Dativ zum Genitiv, Akkusativ oder Nominativ. Die Kasusform hingegen betrifft die einem gewählten (selektierten) Kasus zugeordnete Endung, so beim Genitiv eines maskulinen oder neutralen Substantivs das Vorkommen oder das Fehlen der Endung -(e)s. Veränderungen gegenüber dem geltenden Standard kommen in beiden Bereichen vor. Ich behandele hier Veränderungen in der Kasuswahl (nach EISENBERG 1999 in der Kasusselektion), nicht

1 Vgl. http://www.korrekturen.de/forum.pl/md/read/id/22928/sbj/wider-besserenwissens/ (27.5.13).

2 In der Grammatik werden traditionell „enge Apposition“ wie König in König Heinrich und lose Apposition wie der neue König in Heinrich, der neue König unterschieden. ENGEL (2004:449) nennt nur letztere „Apposition“.

3 EISENBERG (1999:74) spricht statt von Kasuswahl von „Kasusselektion“. 
Veränderungen in der Kasusform (vgl. dazu VATER 2008), jedoch treten, besonders im Plural, oft beide Vorgänge kombiniert auf. ${ }^{4}$

\section{Kasus in Koordinationen}

\subsection{Vorbemerkungen}

Normalerweise stehen alle koordinierten Nominalphrasen (NPs) im gleichen Kasus. Konjunktionen wie und / oder sind verbindende Elemente, nicht aber Regenzien: Die verbundenen Konstituenten werden von anderen Elementen, z.B. Verben und Präpositionen regiert. Durch Konjunktion verbundene Konstituenten müssen kasusgleich sein. So verlangen die Verben sehen und beobachten den Akkusativ; die Präposition wegen verlangt den Genitiv, die Präposition auf den Dativ bei Ruhelage, den Akkusativ bei Richtungsangaben. Erwartbar wäre also etwas wie Ich beobachtete einen alten Mann und eine Frau mit einem Hund und nicht: ... einen alten Mann und einer Frau mit einem Hund, oder wegen des Regens und des starken Winds, und nicht wegen des Regens und dem starken Wind (vgl. 2-10 bis 2-14).

Besonders auffällig ist die Unsicherheit im Zusammenhang mit der Präposition von. Man würde in einem Zeitungsbericht etwas erwarten wie: die Leichen von Generalbundesanwalt Siegfried Buback [...] und seinem Fahrer Wolfgang Göbel; stattdessen fand ich in einer Mitteilung im Kölner Stadtanzeiger: die [...] Leichen von Generalbundesanwalt Siegfried Buback [...] und seines Fahrers Wolfgang Göbel (vgl. 2-19). Die gleiche Unsicherheit findet sich auch in (losen) Appositionen, vgl. z. B.: ein musikalisches Porträt von Salomo, des israelischen Königs (Klassikforum, WDR 3, 3.1.2007, 11.15 Uhr). Offenbar sehen viele deutsche Sprecher und Schreiber von + Dativ als Genitiversatz an und beeilen sich dann, diesem „falschen Genitiv“ in der Apposition oder Koordination einen „richtigen Genitiv“ folgen zu lassen (vgl. VATER 2006, 2007).

Der hier dokumentierte Kasuswechsel ist zweifach zu verstehen: synchron, als Veränderung innerhalb eines (koordinierten) Syntagmas, und diachron, als sich über einen gewissen Zeitraum erstreckender Vorgang zur Umwandlung des deutschen Kasussystems (möglicherweise sogar als dessen Untergang).

$4 \quad$ Bzw. ist es oft nicht entscheidbar, ob der Sprachproduzent unsicher in der Kasuswahl oder in der Kasusform ist (vgl. 2-06, 2-32 und 2-58). 
Ungleiche Paare: Kasuswechsel in koordinierten Nominalphrasen

\subsection{Genitiv + Dativ}

Ein Substantiv regiert innerhalb einer NP eine Genitiv-NP: der Leiter des Instituts. Auch hier findet man Verstöße, wenn ein $\mathrm{N}$ eine koordinierte NP regiert. Oft wird eine Genitivphrase mit einer Dativphrase koordiniert. ${ }^{5}$ In einigen Fällen wird der Wechsel zum Dativ offenbar dadurch ausgelöst, dass eine oder mehrere feminine Genitiv-NPs vorangehen, denn bei Feminina tritt Synkretismus zwischen Genitiv und Dativ (wie auch zwischen Nominativ und Akkusativ) auf. Dies belegen (2-01)-(2-04).

(2-01) Als Metapher historischer Erinnerung und als Chiffre menschlicher Existenz und seinen Lebenswegen aber zählt das „Mahnmal für die ermordeten Juden Europas“ zu den besten Kunstwerken, die [...]. (G. IMDAHL: Verloren im Raum. In: KStA, 16.12.04:28)

(2-02) Von Anf. April bis Ende Mai 1896 lebte sie mitten in Moabit: In der Perleberger Str. 23 in der Wohnung ihrer Tante Paula Rabe, ihrem Patenonkel_ Louis_ und deren Tochter Ella. (SABINE KRUSEN: Ich lebe jetzt ganz mit den Augen. Die Malerin Paula Modersohn-Becker in Berlin-Moabit. In: ecke turmstraBe, Febr. 2012:10)

(2-03) Das Haus versteht sich zudem ... als ständige Vertretung der fernen Schwabenheimat, der Pflege und dem Hörgenuss_ seiner Sprache und dem Erhalt_ der heimatlichen Identität. (http:// www.artenschutztheater.de/uebersicht_01.php, 1.10.10)

(2-04) Fahrt durch das malerische Elbetal und Isertal nach Harrachov, Besichtigung der Glashütte und dem Glasmuseum. (Traumhaftes Riesengebirge. In: BLZ, 20./21.8.10:Reise P3)

Doch finden sich Abweichungen nicht nur nach vorangehender femininer Genitivphrase, wie die restlichen hier angeführten Beispiele zeigen. Offenbar wird der Dativ von vielen Deutschsprechern als ,eine Art Genitiv' empfunden, weil im ,Genitiversatz' des Typs das Haus von meinem Vater (statt: meines Vaters) oder gar meinem Vater sein Haus ein Dativ vorkommt:

(2-05) [...] verbreitete Ibrahim R. [...] zahlreiche Audio- und Videobotschaften des El-Kaida-Gründers Osama Bin Laden und dessen Stellvertreters Eiman al-Sawahiri und dem inzwischen

5 In den Belegen wurde jeweils die abweichende Kasusendung (bzw. die Leerstelle bei mangelnder Kasusendung) unterstrichen. 
getöteten Terrorführer_ Abus Mussab el Sarkawi. (Mutmaßlicher Bin-Laden-Helfer. In: KStA, 11.10.06:5)

(2-06) Nach Auswertung aller 94 Space-Shuttle-Missionen sowie zehn Flügen mit russischen Sojus-Raumkapseln [...] sei [...] aufgedeckt worden, [...]. ${ }^{6}$ (Nasa: Keine betrunkenen Astronauten. In: KStA, 30.8.07:19)

In (2-07) steht der unangebrachte Dativ zwischen zwei standardgemäßen Genitiven:

(2-07) Die Karriere von Anne Sophie Mutter führt sie durch alle bedeutenden Musikzentren Europas, den USA und Asiens. (germanwings Magazin 1/2007:18)

Viele Deutschsprecher kommen auch bei der Koordination einer Singularmit einer Plural-NP ins Schleudern:

(2-08) Währenddessen hockten die Vermittler wieder einmal [...] mit den Entführern zusammen. Um die letzten Details für die Freilassung der neun Deutschen, vier Schweizer und einem Niederländer_auszuhandeln. (R. SCHULZE: Chrobogs Zwischenstop. In: KStA, 20.8.03:2)

(2-09) angesichts der anhaltenden Kämpfe und einem Klima_der Angst (Nachrichten, Kulturradio RBB, 12.3.12:15.00 Uhr)

(2-10) Die Börsen sind ohnehin wegen der Schuldenkrise und den Sorgen vor einem Konjunktureinbruch verunsichert. (ANNA SLEEGERS: Deutsche Bank reißt Dax in die Tiefe. In: BLZ, 6.9.11:1)

In (2-10) zeigt sich gleichzeitig eine andere Tendenz: Die Häufung von Dativendungen nach wegen hängt damit zusammen, dass bei dieser Präposition schon seit längerem die Rektion zwischen Genitiv und Dativ schwankt (vgl. dazu Duden, Grammatik 2006:619). Bei wegen nimmt die in der Umgangssprache schon lange übliche Dativ-Rektion mittlerweile auch in der Standardsprache sehr zu, so dass hier das Problem der grammatisch richtigen Koordination und das Problem der Rektion von wegen zusammen auftreten, was sich in einer Vielzahl ungrammatischer wegen-Koordinationen manifestiert; hier weitere Belege, wobei die Mord-Beispiele (2-12) und (2-13) be-

$6 \quad$ Hier ist standardsprachlich von + Dat statt Gen zugelassen, da Gen bei NPs im Plural ohne Det oder Adj nicht als solcher erkennbar ist (vgl. Duden 2006:979f.); zu erwarten wäre demnach: von zehn Flügen. Möglicherweise handelt es sich hier auch um falschen Bezug des zweiten Koordinats auf die Präp nach. 
sonders auffällig sind, weil hier in beiden Konjunkten das gleiche $\mathrm{N}$ auftritt, einmal mit dem vom Standard geforderten Genitiv, einmal mit dem vom Standard abweichenden Dativ:

(2-11) Der frühere peruanische Präsident Alberto Fujimori ist [...] wegen Mordes, schwerer Körperverletzung und dem Verschwindenlassen_ von Personen angeklagt worden. (KStA, 7.9.2001:6)

(2-12) Wegen dreifachen Mordes, versuchtem Mord, gefährlicher Körperverletzung [...] muss sich [...] der 29-jährige Marco M. vor dem Landgericht in Limburg verantworten. ${ }^{7}$ (T. MORCHNER: Erdrückende Beweise. In: KStA, 16.4.07:14)

(2-13) Sie verurteilte ihn wegen dreifachen Mordes und einem Mordversuch_zu einer lebenslangen Haftstrafe. (T. MORCHNER: Ein Mörder. In: KStA, 5.6.07:18)

Verwunderlich ist die Verwirrung bzw. Abweichung vom Standard im Kulturprogramm des Radiosenders WDR 3, wo man so etwas wirklich nicht erwartet. Würde der Sprecher (bzw. der Autor der Vorlage) vielleicht auch (im Nominativ) den derb-umgangssprachlichen Genitiv-Ersatz (vgl. meinem Vater sein Haus) gebrauchen und sagen der Wiener Philharmoniker ihr unnachahmlicher Sound?

(2-14) [...] wegen der Wiener Philharmoniker und ihrem unnachahmlichen Sound_. (Mosaik. WDR 3, 23.2.2008:9:25 Uhr)

Es gibt Fälle, in denen die Koordination einer Genitiv-NP mit einer Dativ-NP als Standard anerkannt wird, nämlich, wenn im Plural eine von wegen regierte NP mit einer ,nackten' NP (d.h. ohne Det oder Adj) verbunden wird: der Angeklagte wurde wegen Mordes und Raubüberfällen angeklagt. ${ }^{8}$

\subsection{Dativ + Genitiv}

Ebenso häufig finden sich jedoch auch Fälle, in denen in einer Koordination ein Dativ mit nachfolgendem Genitiv kombiniert wird. Auch hier spielt die

$7 \quad$ Hier gibt es zwei Standardoptionen: wegen Mords oder wegen Mordes (vgl. wegen dreifaches Mordes in 2-12).

$8 \quad$ Nach Duden (2007:762) wird wegen ,bei stark flektierten Substantiven im Plural standardsprachlich nur mit dem Dativ verbunden, wenn der Genitiv formal nicht zu erkennen ist [...] wegen Geschäften verreist [...].“ Hier ist anzumerken, dass diese Regelung (vgl. RGD 985) auch für nackte NPs im Singular gilt: wegen Nebel; allerdings ist hier auch noch weiterhin Genitiv möglich: wegen Umbau(s) gesperrt. 
mangelnde Kasusdifferenzierung femininer NPs als Auslöser für einen falschen Dativ-Anschluss eine Rolle:

(2-15) [...] seiner Vergangenheit huldigen kann und natürlich ihrer Helden [...] (Reporter im heute-Journal. ZDF, 13.12.11:22.10 Uhr)

(2-16) Sie resultiert aus der Endlichkeit der Speicherkapazität im menschlichen Gehirn und der heute schier unendlichen Möglichkeiten, auf Wissen zurückzugreifen. (PAULINE KREBS: Der Computer allein macht nicht dumm. In: BLZ, 15.11.12:12)

Besonders charakteristisch ist die Koordination einer von-Phrase mit einem Genitiv. Hier liegen die gleichen Gründe vor wie bei dem in 2.1 erörterten Fall mit umgekehrter Reihenfolge (Gen + Dat): Der Dativ bei der Präposition von wird als „eine Art Genitiv“ (Genitiversatz) aufgefasst; die Sprecher beeilen sich, ihm einen „richtigen Genitiv“ folgen zu lassen (vgl. VATER 2006, 2007):

(2-17) Am Mittwochmittag trafen sich Vertreter von Polizei, des Parkhausbetreibers und der Stadtverwaltung zu einem Krisengespräch. (T. MORCHNER: Domparkhaus. In: KStA, 26.10.06:33)

(2-18) Der Leuchtturm von Nidden, Arbeitsplatz von Irina Alexandrowna und ihres Mannes [...]. (Auf der Kurischen Nehrung. NDR, 15.3.07:20.55 Uhr)

(2-19) Tatort Karlsruhe: Die zugedeckten Leichen von Generalbundesanwalt Siegfried Buback (hinten) und seines Fahrers Wolfgang Göbel. (Bildunterschrift im KStA, 24./25.3.07:3)

(2-20) Immerhin liegt die Gegend ungefähr auf dem Breitengrad von Nowosibirsk, des nördlichen Baikal und des Südzipfels von Kamtschatka. (LACHAUER 2003:59)

(2-21) Mubaraks Stellvertreter Omar Suleiman traf sich am Wochenende mit Vertretern von Demonstranten und der verbotenen Muslimbrüder_. (JUliA GERLACH: Erste Gespräche mit der Opposition. In: BLZ, 7.2.11:1)

(2-22) Danach sind künftig rechtsextreme Kundgebungen zur Verherrlichung von Wehrmacht und anderer NS-Organisationen [...] untersagt. (Aufmärsche verboten. In: KStA, 26.10.06:10)

(2-23) Die Beseitigung der Zarenherrschaft stand bereits lange vor Ausbruch des Ersten Weltkriegs auf der Agenda von Alexander Helphand-Parvus und seiner marxistischen Mitstreiter_. (Leserbrief GÜNTER ZEMELLA. In: Der Spiegel, 17.12.07:8) 
Ungleiche Paare: Kasuswechsel in koordinierten Nominalphrasen

(2-24) Aber auch Manager von Bosch, ABB und des schwedischen Stromkonzerns Vattenfall sind jetzt im Gespräch. (Lead zu: Poker um Chefposten bei Siemens. In: KStA, 7.5.07:9)

(2-25) [...] erklärte ein Sprecher [...] vor einem überlebensgroßen Plakat mit den Gesichtern von Benazir Bhutto und ihres Vaters Zulfikar. (WILLI GERMUND: Benazir Bhuttos Nachfolge. In: KStA, 31.12.07:7)

(2-26) Frankreich hat seit Jahresbeginn rund 11000 Roma nach Rumänien und Bulgarien abgeschoben [...]. Dies rief auch harsche Kritik von Menschenrechtsgruppen, der Kirchen und der Vereinten Nationen hervor. (THORSTEN KNUF: EU fühlt sich von Sarkozy betrogen. In: BLZ, 15.9.10:6)

(2-27) Der Besuch von Kindergärten und Schulen dient zur Verbreitung von Kenntnissen über Biologie und eines besseren Verständnisses von Natur. ${ }^{9}$ (Spektakuläre Flugschauen mit Greifen. In: Pulheimer Nachrichten - Wochenende, 8.8.07)

Einen schlimmen Patzer habe ich in einem Programmheft der Kölner Philharmonie entdeckt, wo nach von ein Dativ mit einer Genitivphrase koordiniert ist, die aber - wohl als Abglanz des Dativs - den Dativ-Artikel dem enthält:

(2-28) Abgesehen vom Finale der 2. Sinfonie, von einigen Liedern und dem anlässlich des deutschen Sieges im DeutschFranzösischen Krieg komponierten Triumphliedes, ist die Akademische Festouvertüre [...]. (Programm zum Konzert in der Kölner Philharmonie, 20.4.07:7)

Während in all diesen Fällen ein von-Attribut (ein ,Genitiv-Ersatz') als Auslöser für den Wechsel zum (,richtigen') Genitiv dient, handelt es sich im folgenden Fall nicht um ein Attribut, sondern um ein präpositionales Objekt (berichten ...v von ...):

(2-29) Zahlreiche Zeugen berichteten [...] von Hinrichtungen auf offener Straße und tödlicher Prügel_ in Haft. (ToM ScHIMMECK: Ein Hauch von Hoffnung für Nigeria. In: BLZ, 3./4.11.12:8)

Im folgenden Beispiel könnte das fehlende Dativ-Plural-n den Wechsel zum Genitiv ausgelöst haben:

$9 \quad$ Die fragliche NP ist Attribut der NP Verbreitung, wird also nicht von der Präp über regiert (in dem Fall wäre Akk angebracht): Kenntnissen über ein besseres Verständnis wäre semantisch abwegig. 
(2-30) Vertreter aus sechs Länder_ [sic!] und des Roten Kreuzes würden zunächst von Venezuela aus in die kolumbianische Stadt Villavicencio fliegen, sagte Chávez in Caracas. (ChávezAktion für Farc-Geiseln. In: FR, 29.12.07:7)

Noch anders liegt der Fall bei (2-31), wo die gewählte Formulierung fälschlich ein (semantisch unsinniges) Attributverhältnis zwischen Vorsitzenden und des ehemaligen Rektors ausdrückt.

(2-31) Sie konnten sich damit jedoch nicht beim Vorsitzenden des Hochschulrats und ehemaligen Rektors der Regensburger Universität, Helmut Altner, durchsetzen [...]. (CHRISTINE BURTSCHEIDT: Zwischen Angst und Aufbruch. In: SZ, 18.6.08:17)

Gemeint ist offenbar eine Koordination beim Vorsitzenden und ehemaligen Rektor; das heißt: Es handelt sich um eine Person, die gleichzeitig Vorsitzender und (ehemaliger) Rektor ist.

\subsection{Akkusativ + Dativ}

Häufig wird eine Akkusativ-NP mit einer Dativ-NP kombiniert, besonders bei durch:

(2-32) Rocchigiani hat seiner sportlichen Karriere durch Alkoholeskapaden, Schlägereien mit Polizisten und unzuverlässigem Verhalten oft mehr geschadet als das Boxmilieu. (G. WALDHERR: Rocky bezwingt ... In: KStA, 23.9.02:36)

(2-33) So wird durch eine sprunghafte, fragmentarische Motivik und einem resignativen Charakter [...], der Anschein von Verzweiflung hervorgerufen. (Programm zum Konzert in der Kölner Philharmonie, 20.4.07:16)

(2-34) Diese Verluste werden durch den Zustrom der Jüngeren und der leicht wachsenden Bevölkerungsgruppe zwischen 50 und 64 allerdings mehr als ausgeglichen. (RAINER RUDOLPH: Köln wird Millionenstadt bleiben. In: KStA, 15.2.08:25)

(2-35) Die Projektgruppe „Stolpersteine“ wird unterstützt durch: die Arbeitsstelle für evangelische Jugendarbeit, den Bund der AntifaschistInnen, dem Historischen Verein zu Frankfurt (Oder) e. V., [...] der Stadtverordnetenversammlung Frankfurt (Oder), [...] , den Zeugen Jehovas und vielen Einzelpersonen, denen diese Form der Erinnungsarbeit ein besonderes Anliegen ist. (www.stolpersteine-ffo.de, 2.9.11) 
Ungleiche Paare: Kasuswechsel in koordinierten Nominalphrasen

(2-36) Durch die vertikale Lastenverteilung entlang des Achsenskeletts und der damit verbundenen Kumulation von Krafteinwirkungen ist besonders die Lendenwirbelsäule beträchtlichen Strapazen ausgesetzt. (Orthopress 2/2001:13)

(2-37) Zu Beginn des 20. Jahrhunderts war die Einwohnerzahl in Berlin $[\ldots]$ durch die schnelle Industrialisierung und dem damit verbundenen Zuzug [...] rapide gewachsen. (WIKIPEDIA: Erlöserkirche Berlin-Moabit, 4.7.11)

(2-38) Thailänder in gelben Hemden streifen durch die Abfertigungshalle und dem weit gespannten Dach des riesigen Glaspalasts an Bangkoks Suvarnabhumi-Flughafen [...]. (WILLI GeRMUND: Ein Proteststurm in Gelb. In: KStA, 27.11.08:3)

Doch findet sich Wechsel vom Akkusativ zum Dativ auch nach anderen Präpositionen, hier belegt bei für, gegen, ohne, über und um:

(2-39) Der Triumph für die Sänger der Hauptpartien, Gerd Brenneis und Janis Martin, sowie für den Dirigenten Heinrich Hollreiser, dem Chorleiter Walter Hagen-Groll und alle Mitwirkenden reflektierte den Sog einer Aufführung, deren Qualität sich immer mehr verdichtet hatte. (In: Der Tagesspiegel, 1.7.80. Kritik der konzertanten Rienzi-Aufführung an der Deutschen Oper, Berlin)

(2-40) Er [Wiefelspütz - H.V.] forderte deshalb Investitionen in doppelte Versorgungssysteme für wichtige Bereiche des öffentlichen Lebens, wie die Stromversorgung, Handy- und Computernetze oder auch die Verkehrsnetze von S- und UBahnen sowie dem Fernverkehr. (Berliner S-Bahn-Polizei fahndet nach Tätern des Brandanschlags. In: GoogleMitteilungen, 25.05.11:09.14 Uhr)

(2-41) Der linksextremistischen Szene gelingt es [...] immer weniger, Anhänger für die alljährlichen Gewaltrituale und dem militanten Kampf in Kreuzberg zu finden. (Kasten „linksextreme Szene“ zum Artikel von A. Kopietz / L. SCHNEDELBACH: Hochgefährliche Mischung. In: BLZ, 8.5.12:15)

(2-42) Baldrian Stark für die Nacht darf nicht eingenommen werden, wenn Sie überempfindlich (allergisch) gegen den Wirkstoff oder einem der sonstigen Bestandteile sind. (Baldriparan, Wiewelhove GmbH, Ibbenbüren, Gebrauchsinformation) 
(2-43) [...] kommt nicht ohne Galgenhumor und einer absurden Komik aus (MARTINA SALEK. In: Kultur aktuell. Kulturradio RBB, 21.11.11:8.45 Uhr)

(2-44) Sie kommt ohne Umfahren der Begrenzungsschilder, Zwischenstopp im Sandkasten oder unfreiwilligem Halt auf der Wiese ins Ziel. (KeRSTIN MÜLLER-SCHLINKE: Familienurlaub ... im Burgenland. In: mampa. Magazin für aktive Familien 4/2007:70)

(2-45) Die Hochschulen haben ihre Lehrinhalte bereits den vergleichsweise kurzen Bachelor-Studiengängen angepasst. Allerdings bleibt unklar, wie dies ohne Überforderung der Schüler und Studenten oder einem Niveauverlust der Abschlüsse umgesetzt werden kann. (Prof. Dr. H. KNOSPE: Leserforum. In: KStA, 7.3.08:22)

(2-46) Von näselnden Dienern über schräge Ganoven, Tantenklamotten und Paukerfilmen, bis hin zum Shakespeare- und Sternheim-Protagonisten. (Programm Januar-März, SchlossparkTheater Berlin 2010:32)

(2-47) Die Wohnung verfügt über vier separate Zimmer, einem gefliesten Wannenbad mit Fenster sowie einer Abstellkammer. (Anzeige im Internet v. Immobilien Klosinski, Mietwohnungen in Charlottenburg, Februar 2008)

(2-48) Diesmal geht es um das Thema Kinderlosigkeit und den dadurch zukünftig fehlenden Rentenzahlern. (Ansage zu einem Hörstück, Kulturradio RBB, 19.10.10:14.10 Uhr)

(2-49) Mir geht es nur um Deinen Antrag und dem für Deinen Vater. (E-Mail Prof. Dr. R. R., Berlin, 29.1.10)

(2-50) Regisseur Thomas Ostermeier lotet Shakespeares Drama um männliche Konkurrenz und latentem Rassismus neu aus. (Bilduntertitel. In: BLZ, 12.10.10:25)

Und erwartungsgemäß findet sich Kasuswechsel auch in der Verbrektion, also bei koordinierten Objekten eines Verbs:

(2-51) Gerichtsmediziner diagnostizierten außerdem Blutspuren im Slip der Toten und einem oberflächlichem Riss an der Scheide. (Alles für die Tochter, BLZ, 3.3.10:28) ${ }^{10}$

10 Wahrscheinlich hat der Schreiber des Satzes die NP einem ... Riss an der Scheide irrtümlich als Attribut zu Blutspuren aufgefasst, was aber keinen Sinn ergäbe 
Ungleiche Paare: Kasuswechsel in koordinierten Nominalphrasen

\subsection{Dativ + Akkusativ}

Auch der umgekehrte Fall, Wechsel vom Dativ zum Akkusativ, kommt vor, wenn auch seltener; hier je ein Beispiel aus der Verb- und der PräpositionsRektion:

(2-52) Sein Hass war abgrundtief, galt dem anderen doch seine geheime Bewunderung: dem genialen Musensohn und linken Schwärmer [...], den erfolgreichen und mit seinem Anderssein kokettierenden Künstler voller Charme und Esprit. (TH. MoLTZAHN: García Lorca. In: KStA, 19./20.8.06:27) ${ }^{11}$

(2-53) Weitere Aufschlüsse erwarten die JVA-Leitung und Polizei von der Obduktion der Leiche und die Vernehmung von Mitgefangenen. (Siegburg. Häftling erhängt sich. In: KStA, 28.4.08:16)

Im folgenden Fall ist schwer zu bestimmen, welcher Kasus vom Schreiber intendiert wurde, da die Numerusendung im Plural von Fremdwörtern oft nicht eindeutig festgelegt ist. Handy und Kamera haben -s-Plural (in allen Kasus), so dass kein Dativ-n auftritt; in Handys und Kameras kann also einwandfrei als Kombination zweier Dative (wie bei in gefordert) aufgefasst werden. Player ist eher mit 0-Plural üblich, der sich dann im Nom, Gen und Akk findet, während beim Dativ -n erwartet wird (vgl. die Bürger, den Bürgern). ${ }^{12}$ So kann man in Beispiel (2-54) annehmen, dass hier Akk intendiert ist.

(2-54) Die Flash-Speicher sind von Speicherkarten in Handys, Digitalkameras oder MP3-Player_bekannt. (SoNJA HöDL: Wechsel bei den Speichermedien. In: SZ, 24.9.07:21)

Bei Koordination zweier Präpositionen, von denen eine den Dativ, die andere den Akkusativ regiert, scheint der Akkusativ zu siegen, wie Google-Belege für mit oder ohne zeigen:

(Blutspuren im Slip ... und einem Riss an der Scheide), wäre dazu grammatisch falsch (in würde fehlen: Blutspuren im Slip ... und in einem Riss ...).

11 Eventuell ließe sich dieser Beleg auch als komplexe NP mit loser Apposition auffassen, doch liegt es nahe, hier eher koordierte NPs anzunehmen (eine Aufzählung der positiven Eigenschaften des Betroffenen). Aber auch bei der Interpretation von den ... erfolgreichen Künstler als Apposition wäre der Akk ungrammatisch.

12 Im Duden-Fremdwörterbuch (2006:167) wird für CD-Player im Plural „-“, also 0 -Plural vermerkt. 
(2-55) Mit oder ohne Glastürme. Pläne für Brückenkopf werden konkreter (Überschrift, Oberösterreichische Nachrichten, Linz, 21.4.10; Google, 22.4.10)

(2-56) Bildungsplanung: Mit oder ohne die Beschäftigten? Projekt KOMPASS erprobt neue Wege (48 BWP 6/2005, M. Allespach PD Dr., IG-Metall-Bezirksltg. Baden-Württemb., Stuttg., H. Novak Dipl. Soz., Projektbüro für innovative Berufsbildung, Heidenheim, 23.4.10)

Einen Kommentar zu „Mit oder ohne wen oder wem?“ findet man im Internet:

Es müsste ... heißen: mit einem Nachfolger oder ohne einen Nachfolger. Da die Wendungen mit oder ohne und mit und ohne häufig vorkommen und ausformuliert sehr schwerfällig klingen, hat sich auch in der Standardsprache eingebürgert, sie mit dem Akkusativ zu verwenden. Da ohne der Wortgruppe näher steht, hat es sozusagen bei der Fallzuteilung gewonnen. (In: Fragen Sie Dr. Bopp, 19.2.10) ${ }^{13}$

Man findet auch bei anderen Präpositionen Koordination mit ,vereinheitlichtem Kasus':

(2-57) Wer in Zahony nicht bei oder für die Bahn arbeitet, lebt irgendwie von der Grenze. (H.-J. DECKERT: Freizügigkeit diesseits der Theiß. In: KStA, 1./2.11.07:10)

Das ist natürlich - nach noch geltenden grammatischen Regeln - ganz falsch. Es kann nur heißen Wer ... bei der Bahn oder für die Bahn arbeitet.

\subsection{Nominativ + Dativ / Akkusativ}

Erstaunlicherweise wird neuerdings ein Nominativ in einer koordinierten NP auch gelegentlich mit einem obliquen Kasus (Dativ oder Akkusativ) verbunden. Hier ein Beispiel für Nom + Dat:

(2-58) Ein Müsli zum Frühstück kann den Wissenschaftlern zufolge das Zeugen eines Jungen begünstigen, ebenso wie Kalium, Kalzium sowie den Vitaminen C, E und B12. Die Studie erschien in der Zeitschrift „Proceedings of the Royal Society.“ (afp. In: KStA, 23.4.08:18)

Das Verb begünstigen fordert einen Subjekt-Nominativ und ein AkkusativObjekt. Beide können aus koordinierten NPs bestehen. In (2-58) kann es sich nur um ein koordiniertes Subjekt handeln (wer oder was begünstigt das Zeu-

13 Vgl. http://canoo.net/blog/2010/02/19/mit-oder-ohne-wen-oder-wem/ (22.4.2010). 
gen eines Jungen?) - so erstaunlich vom Inhalt her dieser Sachverhalt auch sein mag; der nach sowie auftretende Dativ macht keinen Sinn. Es kann nur heißen sowie die Vitamine $C$, E und B12 (die wohl auch das Zeugen eines Jungen begünstigen). ${ }^{14}$

In allen weiteren Beispielen wird ein Nominativ mit akkusativischen NPs verbunden:

(2-59) Da packt einen das olympische Gefühl und den olympischen Geist. (ANDREAS BITTNER: ZDF Olympia Highlights, 22.8.08: 20:40 Uhr)

(2-60) Denn die Sängerin hatte bei Vertragsabschluß auf eine Zusatzvereinbarung gedrängt: eine „notenfeste Kapelle“ und einen Umkleideraum sollten ihr vertraglich zugesichert werden. (In: KStA, 13.9.91:12)

In (2-60) ist der Schreiber möglicherweise vom bereits gewählten Passiv gedanklich ins Aktiv hineingerutscht (... einen Umkleideraum sollte man ihr vertraglich zusichern). Dass deutschsprachige Schreiber sich nicht scheuen, einen Nominativ mit einem Akkusativ zu kombinieren, zeigt auch folgendes Beispiel, bei dem es sich allerdings nicht um eine Koordination, sondern um eine (lose) Apposition handelt; auch hier kann Verwechslung von Aktiv und Passiv vorliegen:

(2-61) In dem noch reichlich bestückten Duty-Free-Shop des Flughafens wird auch Schinken angeboten, polnischen natürlich. (In: KStA 9.3.82:3)

\subsection{Dativ / Akkusativ + Nominativ}

Nicht selten kommt auch der umgekehrte Fall vor, dass ein angestammter, d.h. vom Verb geforderter Dativ bzw. Akkusativ durch einen Nominativ ersetzt wird. Von den beiden Belegen stammt der erste (Koordination von Dat und Nom) aus der mündlichen, der zweite (Koordination von Akkusativ und Nominativ) aus der geschriebenen Sprache.

(2-62) Das kann man nicht vergleichen mit den Staudämmen in der Südosttürkei und [Pause] der Assuan-Staudamm. (FELIX PIRSON, Deutsches Archäologisches Institut: Die antike Stadt Allianoi versinkt im Wasser, Kulturradio RBB, 23.2.11:8:30 Uhr)

14 Möglicherweise liegt auch eine Verwechslung der Präp neben (die Dat fordert) mit der Konjunktion sowie vor. 
(2-63) Drei Figuren hat diese Familien- und Heimatchronik: Vater Pankraz, Sohn Semi und der Gasthof. (DIRK PILZ: Mit der Axt durch die Heimat. In: BLZ, 25.10.11:23)

Auch hier finden sich Parallelen zur Kasussetzung in Appositionen: So erhält in (2-64) eine Dativ-NP eine Apposition im Nominativ (in allen drei koordinierten NPs):

(2-64) Sechs Wochen zelteten die Aktivisten [...] auf einer Obstwiese - das Basislager für die täglichen Waldexkursionen. (Die Waldhüter. In: Greenpeace-Nachrichten 2/2012:17)

In losen Appositionen kommt der Nom statt dem mit dem Antezedens identischen Kasus sehr häufig vor (vgl. VATER 2006). ${ }^{15}$ Darüber hinaus habe ich viele Belege für Nominative in der Verb- und Präpositionsrektion gefunden, die für angestammte (vom Verb bzw. der Präposition geforderte) Dative, Akkusative und sogar Genitive eingetreten sind. So findet sich Nominativ nach Präposition bei grafisch nicht markierten Eigennamen:

(2-65) Dr. Andreas Hördt, wissensch. Mitarbeiter im Geologisches Institut der Universität Bonn (Kölner Universitäts-Journal 4/2001:48)

ZIFONUN et al. (1997/2:1297) konstatieren im Anschluss an JAKOBSON (1971:33), dass in Unsicherheitsfällen die ,allein dem Nominativ vorbehaltene Funktion als merkmallose Form für die Nennfunktion der Rede“ eintritt. Zugelassen vom Duden (RGW 2007:98) sind Nominalphrasen als enge Appositionen im Nominativ bzw. ohne Endung:

Vor allem Namen werden häufig als Apposition an ihren Bezugsausdruck angeschlossen. Dadurch entsteht eine kompaktere Konstruktion, als wenn ein Genitiv- oder Präpositionalattribut verwendet wird. Der Name oder die Bezeichnung erhält dann keine Endung, die den Kasus anzeigt: der Fall Dreyfus, [...] Medizinische Hochschule Hannover [...], Magistrat Berlin [...].

Solche „kompaktere Konstruktionen“ finden sich jetzt häufig, auch wo die gesamte Appositionsphrase keine feste Bezeichnung (wie in Magistrat Berlin) bildet:

15 HeIDOLPH et al. (1981:290) haben diese Tendenz beobachtet und sehen sie offenbar bereits als regulär an: „Appositionen heißen traditionell solche Attribute, die entweder mit dem Kern der SbG [SbG = Substantivgruppe, d.h. Nominalphrase - H.V.] im Kasus übereinstimmen oder - unabhängig von dessen Kasus - im Nominativ stehen.“ 
(2-66) Weiter führt der Bummel durch die Altstadt, in Richtung Alter Markt. (NELLE BäR: Waschküche Berlins. In: BLZ, 21.4.10: Blickpunkt 5)

Die Erklärung als Nominativ in seiner Funktion als Nennform - obwohl dieser Kasus nach Präposition nicht erwartbar ist - ist wohl vorläufig die plausibelste. WIEDENMANN (2004:346) verweist auf die von SAPIR (1921) beobachtete Drift zum invariablen Wort. SAPIR (1921:168) erörtert in allen Sprachen der Welt beobachtbare Tendenzen (,drifts“):

The drift toward the abolition of most case distinctions and the correlative drift toward position as an all-important grammatical method are accompanied, in a sense dominated, by the last of the three major drifts that I have referred to. This is the drift toward the invariable word.

Der Nominativ als die neutrale, invariable Grundform eines Substantivs scheint demnach anzufangen, die obliquen Kasus, die durch ihre Kasusendungen gegenüber der Grundform verändert sind, zu verdrängen. Hier sind weitere Untersuchungen koordinierter NPs notwendig, um festzustellen, ob der Nominativ auch in diesem Bereich an Boden gewonnen hat.

\section{Fazit}

Der untersuchte Grammatikbereich - Koordination deutscher NPs - offenbart starke Veränderungen im Kasusgebrauch, wie ich sie bereits vorher auch in der Rektion von Verben und Präpositionen und in losen Appositionen vorgefunden hatte:

- Genitiv wird oft durch Dativ ersetzt (wie Sick 2006 und 2005 feststellte).

- $\quad$ Ebenso oft wird Dativ durch Genitiv ersetzt (was Sick total entgangen ist).

- $\quad$ Akkusativ wird oft durch Dativ ersetzt.

- Dativ wird gelegentlich durch Akkusativ ersetzt.

- Die drei obliquen Kasus werden häufig durch den Kasus rectus (den Nominativ) ersetzt.

Außerdem macht sich die Tendenz bemerkbar, bei Präpositionen, die verschiedene Kasus (Dat und Akk) regieren, den Kasus zu vereinheitlichen; das ist besonders bei mit und ohne der Fall, wo der Akkusativ siegt, da er von der unmittelbar vorangehenden Präposition ohne gefordert wird: mit und ohne den Deckel. 
SICK (2006) hat in seinem populärwissenschaftlichen Buch (und den FolgeBänden) nur einen Teil der im Deutschen vorgehenden Veränderungen im Kasusgebrauch erfasst. Es ist nicht nur so, dass der Dativ den Genitiv verdrängt, sondern der Genitiv verdrängt auch den Dativ, der Dativ verdrängt den Akkusativ und dieser verdrängt den Dativ, und alle drei können vom Nominativ verdrängt werden. In allen untersuchten Bereichen lässt sich große Unsicherheit im Kasusgebrauch feststellen. In einem Artikel in der Zeitschrift Die Zeit vermutet KRISCHKE (2006:71): „Danach wird die Sprache Kleists und Manns in wenigen Jahrzehnten die meisten ihrer Wortendungen verloren haben, komplizierte Flexionen sind bis dahin verschwunden, viele Grammatikregeln radikal vereinfacht.“

Die in diesem Aufsatz angeführten Beispiele für Kasusvermischung, gelegentlich auch Kasusaufgabe (bzw. Ersetzung durch Nominativ in seiner Funktion als Nennform), stützen die in dem Zeit-Artikel ausgedrückten (wenn auch teilweise spekulativen) Prophezeiungen zu morphosyntaktischen Tendenzen im gegenwärtigen Deutsch. Anders als KRISCHKE (2006:71) spricht EISENBERG (1998:152) vorsichtig von einer „Tendenz [...] in Richtung auf eine weitere Angleichung der Kasusformen“, meint aber: „Das bedeutet aber nicht unbedingt, dass die Kasus ganz verschwinden.“ Immerhin geht Krischke nicht so weit wie KRÄMER (2000), der behauptet (wie in PAULWITZ 2010 zitiert), Deutsch verkomme zur „Schimpansensprache“.

Festzuhalten ist auf jeden Fall, dass seit einiger Zeit eine große Unsicherheit im Kasusgebrauch erkennbar ist, auch bei Journalisten, die für Zeitungen und Zeitschriften mit anerkannt hohem Standard (wie FAZ, Die Zeit und Der Spiegel) schreiben, sowie in der Belletristik, der wissenschaftlichen Literatur und natürlich in Gebrauchsanweisungen etc. Die erkennbaren Änderungen betreffen auch die gesprochene Sprache, wie die angeführten Beispiele aus Rundfunk und Fernsehen belegen, wenn mir hier auch weit weniger Material zur Verfügung stand.

Die nicht nur im hier behandelten Bereich, der Kasussetzung in Koordinationen, sondern auch in der Rektion von Verben und Adjektiven und besonders in losen Appositionen feststellbaren Abweichungen vom standardisierten Kasusgebrauch könnten darauf hindeuten, dass das Deutsche begonnen hat, Kasusmarkierungen völlig aufzugeben, ein Vorgang, der in den anderen germanischen Sprachen (außer dem Isländischen) bereits stattgefunden hat (vgl. hierzu auch die allerdings sehr zurückhaltende Formulierung in EISEN- 
Ungleiche Paare: Kasuswechsel in koordinierten Nominalphrasen

BERG 1998:152). Kontinuierliche weitere Untersuchungen anhand reichlichen Materials müssen diese Tendenzen intensiv verfolgen. ${ }^{16}$

\section{Abkürzungen grammatischer Termini}

$\begin{array}{llll}\text { Nom } & \text { Nominativ } & \text { Adj } & \text { Adjektiv } \\ \text { Gen } & \text { Genitiv } & \text { Det } & \text { Determinans } \\ \text { Dat } & \text { Dativ } & \text { NP } & \text { Nominalphrase } \\ \text { Akk } & \text { Akkusativ } & & \end{array}$

\section{Quellen (auch als abgekürzte Belegangaben) ${ }^{17}$}

$A R D=$ Arbeitsgemeinschaft der öffentlich-rechtlichen Rundfunkanstalten der Bundesrepublik Deutschland. Das Erste (Fernsehsender).

$B L Z=$ Berliner Zeitung. Berlin.

Der Spiegel. Das deutsche Nachrichten-Magazin. Hamburg.

Die Zeit. Wochenzeitung. Hamburg.

ecke turmstraße. Seit Februar 2011 erscheinende Zeitung, hrsg. vom Fördergebiet Aktives Zentrum Turmstraße. Berlin.

$F A Z=$ Frankfurter Allgemeine Zeitung. Frankfurt (M.).

$F R=$ Frankfurter Rundschau. Frankfurt (M.).

Fragen Sie Dr. Bopp! http://canoo.net/blog/2010/02/19/mit-oder-ohne-wen-oderwem/ 12:14 Uhr (Autor: Dr. Bopp; 22.4.2010).

germanwings Magazin 1/2007.

Greenpeace-Nachrichten 2/2012. Hamburg.

HUPPERTH, WALTER: Leserbrief an den KStA, 2.2.2008. http://www.korrekturen.de/ forum.pl/md/read/id/22928/sbj/wider-besseren-wissens/.

Kölner Universitäts-Journal, hrsg. von der Universität zu Köln.

KStA = Kölner Stadtanzeiger. Kölnische Zeitung seit 1802. Köln.

Kulturradio RBB. Rundfunk Berlin-Brandenburg.

16 Ich danke Frau Dr. Nora Wiedenmann, München, für die sehr gründliche Durchsicht des Manuskripts und ihre Hinweise auf formale Unebenheiten.

17 Selbsterklärende Angaben wie Gebrauchsinformationen für Medikamente (z.B. Baldiparan) oder Programmhefte für Konzertprogramme werden hier nicht noch einmal aufgeführt. 
Heinz Vater

Lachauer, Ulla ( $\left.{ }^{7} 2003\right):$ Die Brücke von Tilsit. Begegnungen mit Preußens Osten und Russlands Westen. Reinbek bei Hamburg.

Mampa. Magazin für aktive Familien. Regensburg.

NDR $=$ Norddeutscher Rundfunk (Fernsehsender).

Orthopress. Publikumsmagazin für Patienten, Ärzte und Therapeuten. Köln.

Pulheimer Nachrichten. Tageszeitung für Pulheim (NRW).

Der Tagesspiegel, 1.7.1980. Berlin.

SZ = Süddeutsche Zeitung. Tageszeitung. München.

$W D R=$ Westdeutscher Rundfunk (Fernsehsender).

WDR 3 = Westdeutscher Rundfunk (Rundfunksender).

WIKIPEDIA: Erlöserkirche Berlin-Moabit, 4.7.11.

www.stolpersteine-ffo.de. Informationen zu Stolpersteinverlegungen durch den Historischen Verein zu Frankfurt Oder (2.9.2011).

$Z D F=$ Zweites Deutsches Fernsehen (Fernsehsender).

\section{Literatur}

Bierwisch, Manfred (1989): Event Nominalizations. Proposals and Problems. In: Linguistische Studien A 194:1-73.

Di Meola, Claudio (2000): Die Grammatikalisierung deutscher Präpositionen. Tübingen (=Studien zur deutschen Grammatik 62).

Duden ( $\left.{ }^{7} 2006\right)$ : Die Grammatik. Unentbehrlich für richtiges Deutsch. Mannheim u. a. (=Duden 4).

- ( $\left.{ }^{9} 2006\right)$ : Das Fremdwörterbuch. Mannheim u. a. (=Duden 5).

- ('2007): Richtiges und gutes Deutsch. Wörterbuch der sprachlichen Zweifelsfälle. Mannheim u. a. (=Duden 9, abgekürzt als RGD).

EIsenberg, Peter (1998): Grundriss der deutschen Grammatik. Bd. 1: Das Wort. Stuttgart.

- (1999): Grundriss der deutschen Grammatik. Bd. 2: Der Satz. Stuttgart.

Engel, UlRICH (2004): Deutsche Grammatik. Neubearbeitung. München.

HEIDOLPH, KARL ERICH et al. (1981): Grundzüge einer deutschen Grammatik. Berlin.

JAKOBSON, ROMAN (1936): Beitrag zur allgemeinen Kasuslehre. In: Travaux du Cercle Linguistique de Prague 6:240-288. Wieder in: JAKOBSON, RomAn (1971): Selected Writings. Bd. 2. The Hague, 23-71.

KRÄMER, WALTER (2000): Diskussionsbeitrag auf der Jahrestagung des Instituts für Deutsche Sprache. Mannheim (zitiert in PAULwITZ, 1).

KRISCHKE, WOLFGANG (2006): Ich geh Schule. In: Die Zeit, 29.6.06:71. 
Ungleiche Paare: Kasuswechsel in koordinierten Nominalphrasen

Paulwitz, Thomas (2010): Wird Deutsch zur Affensprache? In: Deutsche Sprachwelt 2010:1.

SAPIR, EDWARD (1921): Language. An Introduction to the Study of Speech. New York.

Sick, Bastian ( $\left.{ }^{1} 2004 /{ }^{29} 2006\right)$ : Der Dativ ist dem Genitiv sein Tod. Ein Wegweiser durch den Irrgarten der deutschen Sprache. Köln.

- ( $\left.{ }^{11} 2005\right)$ : Der Dativ ist dem Genitiv sein Tod (Folge 2). Neues aus dem Irrgarten der deutschen Sprache. Köln.

VARWIG, CORNELIA (2010): Was wird aus unserer Sprache? In: Bild der Wissenschaft 2:64-71.

VATER, HeINZ (2006): Kasus in Appositionen. In: LYLIA. Lyon Linguistique Allemande 8:1-18

- (2007): Kasusgebrauch im gegenwärtigen Deutschen. In: Estudios Filológicos Alemanes 14:11-48.

- (2008): Veränderungen der Kasusformen im gegenwärtigen Deutschen. In: Jahrbuch der ungarischen Germanistik 2007:252-286.

WiedenManN, NoRA (2004): Versprechen - oder der Verlust des Genitiv-s im Deutschen als einsetzender Sprachwandel? In: Estudios Filológicos Alemanes 4:337-374.

ZifonUN, Gisela et al. (1997): Grammatik der deutschen Sprache. Bd. 1-3. Berlin/New York. 